\title{
Le vaudeville dans les nouvelles formes théâtrales foraines : privilège et censure
}

Fanny Prou, Université de Nantes

Au début du dix-huitième siècle, à Paris, se développe sur les foires de Saint-Germain et de Saint-Laurent une nouvelle forme de spectacle utilisant des airs populaires ou popularisés appelés vaudevilles : l'opéra-comique. L'utilisation de ces airs chantés sur de nouvelles paroles est un élément marquant dans l'évolution du répertoire forain. Le théâtre de la Foire a été bien souvent dénigré, vu comme un théâtre bas, grossier, et l'on a tendance à réduire les vaudevilles à des outils comiques ou satiriques. Paul Deslandres explique ainsi le caractère éphémère, d'après lui, de ce théâtre :

Qu'a-t-il donc manqué au théâtre de la Foire pour avoir l'immortalité ? Ce n'est pas tant le goût et la délicatesse que la profondeur. La pochade, le vaudeville amusent pendant une soirée, rarement davantage : demain, nous l'aurons oublié, car nous en aurons vu un qui vaudra le précédent. Ce qui n'est bâti que sur la gaieté ne vit pas longtemps, parce que tout est dans le jeu des interprètes. [...] les forains ont travaillé très vite, pour assurer le pain du lendemain : mauvaise condition pour laisser des chefs d'œuvre. (1)

L'écriture de pièces en vaudevilles ne serait donc qu'un amusement à durée limitée, et surtout, un travail alimentaire. Mais n'est-il qu'un simple objet au service du comique et du divertissement pour les auteurs forains ? Afin de répondre à cette question, qui devrait nous permettre d'élargir cette définition quelque peu réductrice du vaudeville, nous souhaiterions montrer la place des vaudevilles et plus largement des pièces en vaudevilles dans la politique théâtrale de la première moitié du dix-huitième siècle. Il nous semble important, dans un premier temps, de redéfinir le vaudeville du premier quart du siècle, et la forme qui en découlera: l'opéra-comique. Nous ne prétendons pas proposer une analyse définitionnelle exhaustive, car tel n'est pas l'objet de cet article, mais simplement d'en énoncer les principales caractéristiques. Pour une étude plus poussée, nous vous renvoyons aux travaux de Loïc Chahine et Bertrand Porot. Une fois ces jalons posés, nous nous pencherons sur ce qui nous semble être une des fonctions primordiales du vaudeville à la Foire : dans les querelles entre théâtres, le vaudeville ne devient-il pas un outil favorisant le contournement des privilèges ? Rappelons qu'alors la Comédie-Française et l'Opéra avaient des privilèges, accordés par l'État, notamment celui d'être les seuls à pouvoir dialoguer en français, chanter, ou danser, ce qui empêchait les autres acteurs de représenter leurs pièces. Nous voulons ainsi montrer qu'au départ, le développement des pièces en vaudevilles est intrinsèquement lié aux 
questions de politique théâtrale et aux interdictions que subirent les forains de la part de la Comédie-Française. Nous verrons que les vaudevilles, dans les prologues ou dans les pièces métathéâtrales, permettront également de dénoncer ces interdictions. Nous nous demanderons enfin si, dans un contexte de surveillance théâtrale, et peu de temps après l'établissement de la censure préalable (1701), le caractère léger du vaudeville et la pratique de l'allusion n'ont pas permis aux forains de rendre la tâche des censeurs moins aisée ou si, au contraire, ces petits airs parfois si subversifs n'attirèrent pas plus encore la foudre des censeurs. Nous étudierons un cas précis, celui de La Rose de Piron.

\section{Le vaudeville et l'opéra-comique au dix-huitième siècle : essai de définition}

Comme le remarque Chahine, «le vaudeville est considéré comme emblématique des spectacles forains » (223). Il suffit d'ailleurs de revenir sur la préface du Théâtre de la Foire de Le Sage et d'Orneval pour s'apercevoir que le vaudeville fait partie intégrante de la définition de ce théâtre : «De plus, ce théâtre était caractérisé par le vaudeville, espèce de poésie particulière aux Français, estimée des étrangers, aimée de tout le monde et la plus propre de toutes à faire valoir les saillies de l'esprit, à relever le ridicule, à corriger les mœurs » (TFLO I 5-6).

Le vaudeville, dans la première moitié du dix-huitième siècle, est en fait une chanson populaire ou popularisée, chantée sur de nouvelles paroles. C'est le cas par exemple de l'air «Quand le péril est agréable », issu de l'Opéra Atys de Quinault et Lully. Voici les paroles originales de ce vaudeville :

Quand le péril est agréable,

Le moyen de s'en alarmer?

Est-ce un grand mal de trop aimer

Ce que l'on trouve aimable ? (Quinault 6)

On pourrait aussi le chanter sur ces paroles extraites d'Arlequin invisible, pièce de Le Sage, représentée à la foire Saint-Laurent de 1713 :

De ce qui s'offre à votre vue,

Seigneur, nous ne voyons rien, nous.

Par ma foi, les yeux des jaloux

Ont souvent la berlue. (TFLO 82)

Certains airs sont issus d'opéras et se popularisent parfois, étant repris par d'autres auteurs dans leurs pièces, jusqu'à faire partie intégrante du répertoire des vaudevilles.

Ce n'est toutefois pas à la Foire que l'utilisation du vaudeville apparait dans les spectacles. À la fin du dix-septième siècle, le vaudeville avait déjà fait son entrée au théâtre 
dans le divertissement final terminant certaines comédies françaises comme c'est le cas, par exemple, dans Monsieur de Pourceaugnac de Molière en 1669. Il n'apparaît alors pas encore dans le corps de l'intrigue, mais fait bien son apparition sur les scènes théâtrales dès cette époque. Le vaudeville concluant les pièces est d'ailleurs toujours présent dans les pièces foraines au dix-huitième siècle. Il s'agit alors d'un air populaire, inséré dans des pièces de théâtre, dans le corps de la pièce mais également dans le divertissement final.

Maintenant que la définition du vaudeville en tant qu'outil musical est posée, il nous semble également important de rétablir une définition des opéras-comiques ou pièces en vaudevilles comme nous l'entendons lorsqu'on étudie le théâtre de la Foire de la première moitié du dix-huitième siècle. Olivier Bara définit ainsi les pièces utilisant des vaudevilles :

Est vaudeville ou en vaudevilles, toute pièce, comédie ou drame, où alternent le parler et le chanter, le théâtre et la chanson, avec couplets et refrains sur des airs connus appelés «timbres »-1'opéra-comique, quant à lui, fait alterner paroles et chants sur une musique originale. Le vaudeville se définit donc par défaut : contrairement au mélodrame, il pratique l'alternance du dialogue parlé et du couplet chanté ; contrairement à l'opéra-comique, ses couplets se chantent sur des mélodies connues. (141)

Bara généralise sa définition de l'opéra-comique. Celle qu'il donne ici ne s'applique en effet qu'aux opéras-comiques qui apparaissent dans la deuxième moitié du dix-huitième siècle et qui utilisent bien des airs originaux. En revanche, sa définition du vaudeville en tant que pièce est similaire à nos opéras-comiques - version première moitié du dix-huitième siècle : ils se composent également d'une alternance de parler et de chanter sur des airs préexistants. Il faudrait donc revoir cette définition des opéras-comiques et la nuancer. Comment et pourquoi naissent et se développent alors ces pièces en vaudevilles ${ }^{1}$ ?

\section{L'émergence de formes spécifiquement foraines en vaudevilles : la guerre contre les privilèges}

a. Rappels historiques

Depuis 1680, la Comédie-Française a seule le droit de représenter des tragédies et des comédies. Un régime de monopole, mis en place sous le règne de Louis XIV, limite ainsi les productions des théâtres non privilégiés. L’Opéra se réserve le droit de chanter, et la Comédie-Française le droit de parler en français. La Comédie Italienne, elle, si elle n'est pas privilégiée, est une grande institution soutenue et reconnue par le Roi. À côté de ces trois grands théâtres, les forains n'ont presque aucun droit, ce qui explique les procès et querelles qui leurs sont intentés. Malgré ces monopoles, les forains attirent le public en masse, et la 
Comédie-Française va alors multiplier les procès et les interdictions, obligeant les forains à inventer des stratagèmes: pièces par monologues lorsqu'ils n'auront plus le droit de dialoguer; pièce par marionnettes, dans lesquelles on retrouve des vaudevilles lorsque les acteurs sont interdits sur scènes, etc. Ces interdictions vont mener les entrepreneurs forains à sous-louer le privilège de l'Opéra, qui leur permettra, dès 1708, d'insérer des vaudevilles dans leurs divertissements. En effet, Catherine Vondreberg avait obtenu l'autorisation d'utiliser musique et danse dans ses représentations : "Alard et la Veuve Maurice [...] prirent des arrangements pour cette Foire et les suivantes avec le sieur Guyenet alors Directeur général de l'Académie Royale de musique, qui, en vertu des lettres patentes accordées par le Roi à cette Académie, leur permit de faire usage, sur leurs théâtres, de changements de décorations, de chanteurs dans les divertissements et de danseurs dans les ballets » (MFP I 74). Ainsi, sans les associer à un spectacle ou à une troupe, et sans même en trouver le nom, on peut considérer ces premières pièces comme les prémisses des opérascomiques, c'est-à-dire comme une forme de spectacle comique, dans lesquels on pouvait trouver des divertissements chantés et dansés. C'est une première forme d'opéra-comique, une première manière de faire, qui sera par la suite développée.

Ceux qui ne pouvaient pas payer cette redevance à l'Opéra, ne pouvaient donc pas utiliser chant et musique sur scène. Les forains inventent alors une nouvelle forme, utilisée à partir de 1710 : les pièces par écriteaux.

Dans ces pièces, ils n'utilisent aucun dialogue, ne chantent pas mais font de la pantomime. C'est le public qui chante les paroles montrées sur des écriteaux. On comprend donc que le vaudeville devient alors un outil indispensable aux forains et une arme de guerre contre les privilèges. Sur le frontispice d'Arlequin roi de Sérendib ${ }^{2}$, de Le Sage, pièce par écriteaux représentée à la foire Saint-Germain de 1713, on voit ainsi deux Amours tenant un écriteau, sur lequel sont inscrites les paroles suivantes :

$$
\begin{gathered}
\text { ARLEQUIN } \\
\text { Air : Monsieur Lapalisse est mort } \\
\text { C'est lui, plaignez ses malheurs, } \\
\text { C'est lui que le sort ballotte. } \\
\text { Reconnaissez-le à ses pleurs, } \\
\text { Encore plus à sa culotte. (TFLO I 43) }
\end{gathered}
$$

Pendant que le public chantait l'air, les acteurs, Mezzetin, Pierrot et Arlequin, devaient représenter une pantomime, et exécuter différents lazzis, ici celui de montrer leur culotte : «Il montre sa culotte d'Arlequin. Mezzetin et Pierrot se font connaître de la même manière » (43). 
Dès 1714, une forme plus aboutie d'opéra-comique se développe : le terme se trouve associé à une troupe, Saint-Edme et Baron en ayant le privilège. Le 26 décembre 1714, ces deux entrepreneurs obtiennent une permission plus ample de l'Académie Royale de musique pour leurs spectacles qui «prirent dans leurs affiches le titre d'opéra-comique » (Mémoires I 166). La première pièce à porter clairement le nom d'opéra-comique est la pièce de Le Sage, Télémaque, en 1715. Le vaudeville, qui n'était au départ qu'un procédé pour donner des représentations malgré les interdictions liées aux privilèges, et utilisé dans ce but, que ce soit dans les pièces par écriteaux ou dans les opéras-comiques, va alors prendre une place plus importante dans la production foraine puisque même lorsque les forains ne subissent pas de procès, le vaudeville continuera d'exister à travers tout le dix-huitième siècle, tout en diminuant dans la seconde moitié du siècle (pour laisser place aux pièces à ariettes). L'utilisation des vaudevilles, au départ, avait donc bien pour fonction de contrer les théâtres privilégiés.

On retrouve l'histoire de ces querelles et évolutions théâtrales en filigrane dans nombre de pièces foraines. Les pièces en vaudevilles permettaient ainsi, par leur contenu même, de combattre les théâtres privilégiés. Dans La querelle des théâtres, de Le Sage et La Font pour la foire Saint-Laurent 1718, les forains se défendent. C'est un prologue allégorique, mettant en scène la Foire, La Comédie Italienne, la Comédie-Française. La Comédie-Française et la Comédie Italienne sont ici présentées comme des furies jalouses et violentes :

\section{Détruisons tous les forains, Auteurs de notre indigence. \\ De nos propres mains \\ Tuons cette engeance. (TFLO III 36)}

On retrouve cette idée dans Le rappel de la Foire à la vie qui fut représentée en septembre 1721 sur le théâtre de Francisque : dans cette pièce, la Foire, qui allégoriquement est morte $^{3}$ (fermeture du théâtre en 1719) revient à la vie. Ce prologue est évidemment un acte de défense de la Foire. L'Opéra affirme ramener la Foire d'entre les morts. L'air des «Trembleurs » est un air sombre et violent. Ici, c'est la Comédie Italienne qui menace la Foire :

C'est moi, fatale ennemie

Que l'enfer a revomie,

C'est moi qui veux de ta vie

Finir les jours trop chéris.

J'ai de rimeurs une clique

Qui sortent de rhétorique, 


\section{De ton Opéra-Comique \\ Ils vont dégoûter Paris. (440)}

Cette façon de représenter les théâtres rivaux est un topos du théâtre forain, mais dont la visée n'est pas uniquement comique et satirique. Le but est bien de discréditer les autres théâtres afin de rallier le public à sa cause.

\section{b. Étude de cas : Momus censeur des théâtres}

Un autre opéra-comique, Momus censeur des théâtres ${ }^{4}$ de Jacques Bailly, représenté à la foire Saint-Laurent de 1725, propose une réflexion sur les différentes comédies. La pièce s'ouvre sur les personnages allégoriques du Caprice et de la Folie. La Folie découvre le Caprice endormi et le réveille. Il lui explique qu'il lisait une pièce du Théâtre Italien, dont la morale l'a endormi. Quant à la Folie, elle est présente pour rassembler les théâtres, par ordre de Momus, afin d'apprendre d'eux ce qu'ils ont joué pendant l'hiver. Cette remarque est une occasion parfaite pour critiquer les pièces italiennes :

Les Italiens, je te jure,

Ont souffert dans telle aventure :

Les pièces qu'ils représentaient

Avaient le succès ordinaire ;

Car sitôt qu'elles paraissaient,

Zeste,

On les voyait tomber par terre. (Bailly I 68)

Momus paraît ensuite, sur l'air «Des rats ». Cette entrée permet de caractériser Momus, dieu de la satire et des fous, puisque la définition attestée d' «avoir des rats » signifie «avoir des caprices, des bizarreries, des fantaisies » (Acad. 1762). L'arrivée de Momus est l'occasion d'une critique des pièces actuellement représentées sur les théâtres. C'est d'abord l'idée que les théâtres ne cherchent plus à plaire au public qui est pointée dans l'air de «Joconde » :

\section{Le public se plaint hautement,}

C'est l'aveu du vulgaire,

Que les théâtres maintenant

Ne cherchent plus à plaire,

Et que l'Italien manquant

De bonnes comédies,

Ne peut attraper son argent

Qu'avec des parodies. (I 72)

L'autre élément de critique qui paraît dans ce vaudeville est le goût prononcé - trop prononcé ? - des théâtres pour les parodies. La Comédie Italienne, notamment, n'était pas en reste. Elle fit représenter, entre autres, Armide, pièce de Bailly également, parodie de l'opéra du même nom, ou encore Les huit Mariamnes de Piron, parodie des Quatre Mariamnes de 
Fuzelier, ou enfin Le mauvais ménage de Le Grand et Dominique, parodie d'Hérode et Mariamne de Voltaire 5 .

La Comédie-Française paraît ensuite accompagnée d'un poète. Le discours est jonché de sous-entendus : «Seigneur, c'est un poète tragique dont j'avais emprunté le bras pour me soutenir, il a manqué trois ou quatre fois de me faire rompre le cou... » (I 75), dit la ComédieFrançaise ; le Caprice répond : «Avec ces messieurs-là vous êtes souvent exposée à faire des chutes fâcheuses » (I 75). On comprend qu'il s'agit en fait d'une transposition de la chute des pièces de théâtre et particulièrement des tragédies à la Comédie-Française sous une forme allégorique, effet de style très fréquent dans la satire des théâtres privilégiés. S'ajoute ensuite une critique de l'actualité, et notamment de la reprise de Mariamne. À propos de cette pièce, tragédie de Voltaire de 1724, on nous apprend dans les Annales dramatiques qu'elle n'eut pas de succès lors de la première représentation (108). Une plaisanterie fit tomber la pièce : « $\mathrm{Ce}$ grand homme nous apprend qu'au moment où l'actrice qui faisait le rôle de Mariamne portait la coupe empoisonnée à sa bouche, une personne du parterre s'écria : La Reine boit! Ce qui occasionna un grand tumulte » (Bailly IV 108). Le dénouement en fut modifié en 1725 et la pièce devint Hérode et Mariamne. Elle eut alors plus de succès. Ce rappel est bien sûr utilisé ici par l'auteur pour insister sur le manque de nouveauté à la Foire. La Comédie-Française ajoute y avoir donné une autre pièce, Le triomphe du temps, qui eut du succès. Momus ironise, alléguant que c'est uniquement la présence du chant et de la danse dans cette pièce qui a permis son succès. C'est ainsi rabaisser la Comédie-Française à ses rivaux. Un argument de défense de la Comédie-Française est enfin l'arrivée d'une actrice nouvelle sur son théâtre, donnant lieu à une autre réplique de Momus : ce n'est que grâce à elle qu'est accouru le public. L'actrice en question est probablement Marie Dupré, femme de QuinaultDufresne, qui « débuta d'abord à Fontainebleau, le 7 novembre 1724 ; elle y fit tant de plaisir que le roi la gratifia d'un habit fort riche à la romaine, de la valeur au moins de huit mille livres; reçue le 17 du même mois; elle parut à Paris dans Andromaque, par le rôle d'Hermione, le 5 janvier $1725 »$ (Mouhy 464).

Cette scène, rappelant ce que doit la Comédie-Française à Molière et Corneille, est un plaidoyer pour la nouveauté, instrument de défense souvent repris par les forains : «Ma foi, sans Corneille et Molière, vous seriez bien mal dans vos affaires » (Bailly I 80). Mais même ces auteurs lassent, pour avoir été encore et encore repris :

Vous donnez, belle Comédie

Si souvent ce fameux auteur, Que chacun se lasse et s'écrie, D'autant qu'on le sait tout par cœur : 
Molière, mes amours

Vous verrai-je toujours? (I 80)

La même critique que pour la Comédie-Française se fait ensuite, lorsque la Comédie Italienne arrive sur scène. Une allusion au Prince travesti de Marivaux qui avait été reprise, est encore une fois l'occasion d'une raillerie. Le public, seul juge, ne la reçut pas bien : «Paris en l'apercevant / Revenir sur la scène / S'écriait à chaque instant / Tant il lui faisait peine » (Bailly I 90). Une note de l'éditeur nous dit par ailleurs qu'« en effet, ses propos fades et languissants ne finissaient pas ».

La Comédie-Française et la Comédie Italienne ont été évincées. C'est la Foire qui arrive désormais. Elle apparaît alors amaigrie, et en colère contre la Comédie-Italienne. La Foire fait à nouveau référence à la querelle des Mariamnes. Elle avait en effet proposé une pièce, Les quatre Mariamnes, parodie de celle de Voltaire, mais la Comédie Italienne a empiété sur son terrain en proposant Les huit Mariamnes, pièce de Piron. La Comédie Italienne, dans cette pièce, utilise des vaudevilles, que la Foire semble considérer comme son privilège exclusif.

La Foire, contrairement aux autres comédies, reçoit un important compliment de Momus : «J'ai pourtant entendu dire que vous aviez fait de grands préparatifs pour attirer le public chez vous » (I 102). Ce n'est donc pas faute d'avoir voulu plaire et proposer des nouveautés qui fit échouer la Foire cette année-là.

Enfin, l'arrivée de Monsieur Vaudeville, fournisseur de pièces pour la Foire, est l'occasion de proposer une esthétique du théâtre forain. Ainsi, la Foire lui demande d'ajouter dans la pièce du bon goût, de la délicatesse, mais aussi beaucoup de «Mirlitons ». On lit même dans cette pièce un «traité » de style pour plaire aux spectateurs. Un auteur doit «faire entrer du badin » (I 73) dans ses jeux. La satire est bienvenue, mais c'est une satire particulière à laquelle Momus engage les auteurs : celle qui fait rire et permet de se moquer de soi-même. Bailly reprend ainsi un topos : le théâtre doit permettre de se corriger de ses défauts, tout en plaisant.

Les prologues en vaudevilles et les pièces métathéâtrales permettent ainsi de mettre en avant les querelles, et par là, de se défendre contre les attaques des théâtres privilégiés et également de proposer une forme d'esthétique théâtrale. La marque de fabrique du théâtre forain reste ainsi le vaudeville, mais l'on voit déjà poindre ce vers quoi pencheront de plus en plus les auteurs de la Foire : un style plus délicat, afin de contrer les critiques les plus ardentes contre la Foire, qui, bien souvent, mettaient en cause la bienséance. 


\section{Le vaudeville, objet de censure : un contenu gênant ?}

Quelles sont les forces du vaudeville face à la censure ? Les forains parvenaient-ils à la contourner, à l'image des interdictions liées aux privilèges ? Quelles sont les limites de cet outil privilégié des forains qu'est le vaudeville ? Enfin, que va-t-on censurer à la Foire ? La bienséance évoquée précédemment est justement au cœur des questions de censure. Mais la connivence entre les acteurs et le public engendrée par l'utilisation d'airs populaires pouvait être un frein pour les censeurs. Nous allons le voir : utiliser certains airs, certains refrains, permet de jouer sur les sens avec le public. Leur utilisation participait en effet au caractère ludique de la représentation : les auteurs jouaient sur le sens des vaudevilles et l'hypotexte de ces derniers ${ }^{6}$. Une typologie permet de classer les différents types de vaudevilles en fonction du sens des paroles nouvelles mises sur le vaudeville ${ }^{7}:$ les paroles peuvent être concordantes avec l'air ; un air à boire aura ainsi des paroles sur le même sujet. Un air dont les paroles sont discordantes pourra, lui, dire l'inverse des paroles (des paroles grivoises sur un air tendre). L'utilisation peut enfin être neutre, c'est-à-dire que les paroles initiales de l'air n'importent pas, et l'auteur ne joue pas sur le sens. Cet usage se fait notamment pour des raisons métriques. Au sein de tous ces vaudevilles, on trouve un certain nombre de catégories : des airs tendres (ou brunettes), des airs à boire, des airs militaires, des airs d'opéra, ou encore des airs cachant un sens grivois.

C'est à cette dernière catégorie de vaudevilles que nous allons nous intéresser. Nous prendrons pour exemple La Rose de Piron. Mais cette pièce, bien que reposant presque exclusivement sur le régime de l'allusion, n’avait pas pour autant évité la censure. Nous tenterons d'en comprendre les raisons.

\section{a. Argument}

Cette pièce est une vaste métaphore autour du jardinage et des fleurs, derrière laquelle on peut lire clairement une allusion à la sexualité. Dans cette pièce à tiroirs (Plusieurs personnages arrivent tour à tour, et se présentent devant un noyau de personnages fixes) un bel esprit vient d'abord proposer une chanson à la jeune fille. Arrive un vieillard, avec une pomme, puis un berger. Celui qui a la préférence sera ce dernier personnage.

Rosette, se croyant seule, chante qu'elle sent quelque chose « frétiller » en elle. Sylvie la surprend et se demande pourquoi elle est réveillée de si bonne heure. Rosette avoue qu'elle commence, à son âge, à avoir du raisonnement et ne souhaite plus garder la maison mais bien aller garder les moutons seule. Elle apprend ensuite à Sylvie qu'elle doit le jour-même prendre ses emplois de bergère : «L'on m'a promis la clef des champs / Dès qu'on verra la rose éclore » (365). Rosette dit encore à Sylvie qu'elle est résolue d'offrir la « rose » au plus 
joli berger qui lui ferait sa cour. La mère de Rosette arrive, souhaite voir la rose et s'étonne qu'elle soit déjà éclose. Rosette propose qu'on la cueille, ce que refuse la mère, qui demande que la rose reste enfermée à double tour pendant qu'elle part chercher l'Hymen : pour pouvoir mettre Rosette en ménage, il faut que la rose soit intacte. Alors que la mère quitte la scène, elle rencontre l'Amour. Il nous apprend que la mère fut très obligeante dans sa jeunesse. Elle lui demande de parler moins fort de crainte que sa fille ne l'entende et ne prenne exemple sur elle. Avant de partir, elle confie Rosette à Colin. Il doit empêcher qu'on ne cueille la rose: la tâche est difficile. Il chante d'ailleurs qu' «On peut chez nous s'introduire / Par derrière et par devant. / Mille gens de toutes sortes / Viendront nous escalader. / Une maison à deux portes / Est difficile à garder » (381). Rosette lui fait des avances qu'il refuse de peur des représailles et lui avoue qu'une troupe de grenadiers tente d'entrer dans le jardin. Colin s'énerve et part en jurant qu'ils n'entreront pas. Rosette rencontre alors un bel esprit. Il parle en déclamant et Rosette est bien déçue lorsqu'elle comprend que ce qu'il s'apprête à lui donner est une... chanson! Alors qu'elle se laisse convaincre par le bel esprit de lui offrir la rose, un vieillard arrive. Il tente de séduire Rosette qui le repousse jusqu'à ce qu'il lui présente une pomme d'or. Pour l'obtenir, Rosette doit mener le vieillard « Auprès de [sa]... jeunette bergère, / Auprès de [son] rosier » (399). Alors qu'elle va accepter la pomme, elle aperçoit un jeune berger et se détourne du vieillard. Le jeune homme demande à Rosette si la rose a déjà été cueillie, ce à quoi elle répond : "Non, non / Mais je l'offrais à ce barbon / Par qui je suis enrichie » (403). Rosette hésite et demande au berger ce qu'il peut lui offrir. Il répond: «Pour toute richesse / Hélas ! je n'ai qu'un talareritatou, / Je n'ai qu'un tendre cœur » (406). Elle choisit le berger. Les deux se jurent fidélité, l'Amour vient et annonce l'arrivée de l'Hymen et de la mère. Ils arrivent à temps, la rose n'a pas encore été cueillie. L'Hymen décide de donner la rose au jeune berger et la pièce se termine sur une musette, quelques danses « légères et galantes », et on danse et chante un branle sur ce refrain : «Fera bon, bon, bon, derirette / Fera bon bondir ses troupeaux » (414).

\section{b. Une ouvre victime de la censure : le vaudeville et ses malines interprétations}

Cette «pastorale comique » en un acte fut représentée en 1744 pour la première fois, à savoir que lors de cette représentation, c'est la pièce non censurée, retouchée par Favart, qui fut représentée. Son succès semble avoir donné raison à Piron. Mais son écriture est donc largement antérieure, puisque Piron l'avait écrite en 1726. À cette époque, elle avait été interdite par la police. Quels étaient les reproches de l'abbé Cherrier, chargé d'examiner la pièce ? À première vue, peu de choses : 
Il n'en est pas de même des autres termes qui sont répandus dans la pièce et qui peuvent faire naître quelques applications dangereuses; ces termes sont rose, jardin, houlette, voir le loup. Je ne crois pas qu'il faille les retrancher par rapport à la malignité dont on peut être affecté, d'autant plus que si on retranche ces mots, ou les phrases qui contiennent ces mots, il faudra retrancher toute la pièce. Scène XII. Vers la fin, j'ai retranché ces mots : jusqu'à la vache du compère panier, dont on parlera à jamais, en disant qu'il n'en faut pas parler, parce que j'ai eu peur de l'application. (Ms. 3534, lettre du 17 février 1726)

Ce sont ces quelques mots qui pouvaient être à double entente, et c'est ce qui fut cause des méfiances de l'abbé. Mais il ne souhaitait pas pour autant l'interdire :

Au reste, plus j'examine la pièce, et plus je la trouve dans les bienséances du théâtre : toutes les malignes interprétations que l'on peut donner à la rose, à la houlette, ne sont que des interprétations : il faut dans les ouvrages s'attacher au sens que les paroles donnent par elles-mêmes, et ne pas s'attacher à la torture, et à la violence que les esprits de travers peuvent donner. (Ms. 3534, Lettre du 17 février 1726)

Ce sont justement les sous-entendus que l'on retrouve dans les airs, dans l'usage de certains mots, qui sont évoqués par l'abbé Chérier. Mais comme lui-même le souligne, il ne faudrait pas interdire une pièce simplement pour des raisons subjectives.

Examinons de plus près les grivoiseries cachées dans les airs et les «interprétations » qui pouvaient en découler. L'air « Une jeune nonnette en s'éveillant » est déjà porteur de sens pour le public. Derrière l'apparente naïveté de ce titre, l'évocation de la jeunesse et de la négation de la sexualité chez les religieux invite déjà, qui plus est dans un vaudeville, à poser la question de la sexualité. L'usage de ce type d'airs permet très souvent les allusions grivoises : les didascalies permettent également d'appuyer le caractère grivois d'un air. Dans un autre passage, on peut ainsi lire « Rosette, d'un petit air fin ». Cette finesse est bien celle de la jeune fille qui s'éveille à la sexualité et l'air qui suit n'est pas anodin. Le voici :

Autre chose qui t'éveille,

Te met la puce à l'oreille :

Ah, tu soupires tout bas !

Conte-nous ci, conte-nous ça,

Là, là, là,

Je ne le redirai pas. (Piron 362)

Il s'agit de l'air «Ramonez-ci, ramonez-ça » (la suite la plus connue de ce vaudeville étant : «Ramonez-ci, ramonez-ça, la cheminée du haut en bas »). Il ne semble pas nécessaire d'expliciter plus avant le sous-entendu lié à l'utilisation de ce vaudeville. Un autre air, très explicite, est utilisé par Piron peu après :

Air : Une perruquière devant $S^{t}$ Mery. À treize ans je pense 
N'être plus enfant :

Déjà je commence

D'avoir du tourlourirette,

D'avoir du lon la derirette,

(Se touchant au front.)

Du raisonnement. (362)

L'effet d'attente créé par les deux vers «D'avoir du tourlourirette / D'avoir du lon la derirette » renforce le sous-entendu et la grivoiserie du propos. Le vaudeville permet donc de pratiquer l'allusion et l'équivoque. Ainsi, le censeur ne peut que difficilement condamner une pièce qui ne fait que sous-entendre les idées. Or, les vaudevilles sont des outils privilégiés des auteurs lorsqu'il s'agit de pratiquer l'allusion. Nous avons enfin procédé à une analyse statistique des airs dans La Rose. Dans cette pièce, trente-neuf pourcent des vaudevilles ont une utilisation plutôt neutre, servant simplement l'avancée de l'intrigue. En revanche, soixante et un pourcent proposent un sous-entendu grivois, dont dix-huit airs sont des tralalas. Benoît de Cornulier désigne ainsi les «syllabes non significatives » dans son article «Les tralalas ou syllabes non significatives illustrées par des chansons vendéennes » (209-220). Un tralala est donc un air utilisant des syllabes non significatives afin d'éluder un mot, ou de sous-entendre une idée, souvent grivoise. On peut donc penser qu'avec une nette majorité d'airs allusifs, la censure était difficile, voire impossible.

Mais les arguments de l'abbé Chérier évoqués plus haut ne semblèrent pas, face à ces grivoiseries, assez forts. La pièce fut en effet interdite. C'est en fait le lieutenant de Police, Hérault, qui la refusa, conforté par l'abbé Radiguet, comme nous l'apprend une lettre de Piron à Maurepas (DTP III 116). Le vaudeville, cette fois, ne suffit pas aux forains à déjouer les interdictions. Peu d'informations subsistent sur la censure des pièces de théâtre à la Foire. Mais le cas de La Rose nous montre bien la difficulté de censurer des pièces fonctionnant sur le régime de l'allusion : l'abbé Cherrier semble en être bien conscient, bien que les autorités supérieures n'en aient pas décidé de même, et bien souvent, la censure à la Foire touchait des questions de mœurs. Arlequin fille malgré lui, en 1711, fournissait déjà un exemple intéressant de censure à la Foire ${ }^{8}$.

\section{Conclusion}

Le vaudeville, à la Foire, avait donc bien plusieurs fonctions : utilisé au départ comme outil pour contrer les interdictions, son contenu permettait aussi aux forains de se défendre contre les attaques des autres théâtres, et, parfois, devenait un outil publicitaire servant la cause foraine. Il leur permettait aussi d'attirer le public grâce à la connivence qui s'établit 
entre acteurs et spectateurs dans les pièces par écriteaux et dans les opéras-comiques. Le public pouvait facilement reprendre en chœur un vaudeville lorsque les forains les y invitaient. Si le vaudeville, en bien des mesures, fut pour les forains un outil privilégié pour déjouer les tours de leurs adversaires, il n'était pas toujours efficace et sa subversivité, comme nous l'avons vu avec la pièce de Piron, pouvait au contraire attirer la méfiance des censeurs. Mais il est également important de rappeler que, bien après les luttes et les interdictions, le vaudeville perdurera et deviendra un outil esthétique. Toutefois, il ne faudrait pas limiter les vaudevilles à ces seuls aspects. En effet, ils possèdent également une fonction dramaturgique - ils permettent de faire avancer l'intrigue, ou au contraire de la ralentir - et l'on pourrait également s'intéresser à l'aspect financier: un compositeur coûte cher, l'utilisation des vaudevilles permettait alors d'économiser. Ces éléments prouvent que la richesse des vaudevilles au dix-huitième siècle ne se limite donc pas à un simple élément de gros comique et de satire, mais plus encore, n'en déplaise à Deslandres, que ces vaudevilles ne furent pas si éphémères et firent la fortune du théâtre français tout au long du siècle. Ce n'est pas Couplégor, le démon des couplets dans L'enchanteur mirliton, qui nous contredira :

Fameux soutien de la Foire [...],

Prodiguez-lui vos lanlaire, Vos Flonflon, vos lanturelu, Et vos vogue la galère,

Ou son théâtre est perdu. (TFLO VI 12)

\section{Abréviations}

DTP : Claude Parfaict et Godin d'Abguerbe. Dictionnaire des théâtres de Paris. Rozet, 17671770, rééd. Genève : Slatkine, 1967.

MFP : [Claude et François Parfaict], Mémoires pour servir à l'histoire des spectacles de la Foire par un acteur forain. Paris : Briasson, 1743.

TFLO : Le Sage et d'Orneval, Le Théâtre de la Foire ou l'Opéra-Comique, contenant les meilleures pièces qui ont été représentées aux Foires de Saint-Germain et de SaintLaurent. Paris : Ganeau, 1724, Pissot, 1728, Gandouin, 1731-1737, rééd. Genève : Slatkine, 1968.

\section{Bibliographie}

Annales dramatiques ou dictionnaire général des théâtres. Paris : Par une société des gens de lettres, 1810, t. VI.

Bailly, Jacques. Théâtre et œuvres mêlées. Paris : Nyon, 1768. 
Bara, Olivier. «La folie du vaudeville face à la raison de la censure sous la Monarchie de Juillet ». juillet 2012. Universidade do Porto : Facultade de Letras, 2013 : 139-162.

Beaucé, Pauline. «Les jeux de mots dans le répertoire des théâtres de la Foire à Paris au XVIII ${ }^{\mathrm{e}}$ siècle ». Enjeux du jeu de mots : perspectives linguistiques et littéraires. Dir. Esme Winter-Froemel et Angelika Zirker. Berlin / Boston : Walter de Gruyter, 2015 : $65-80$.

---. Parodies d'opéra au siècle des Lumières, évolution d'un genre comique. Rennes : PU de Rennes, 2013.

Campardon, Émile. Les spectacles de la foire. Paris : Berger-Levrault, 1877.

Chahine, Loïc. «Louis Fuzelier, le théâtre et la pratique du vaudeville : établissement et jalons d'analyse d'un corpus », thèse de doctorat, dir. Françoise Rubellin, U de Nantes, 2014. Non publiée.

Cornulier (de), Benoît, «Les tralalas ou syllabes non significatives illustrées par des chansons vendéennes ». Chansons et mémoire, mémoire en chansons : hommage à Jérôme Bujeaud. Dir. A.-M. Despringre. Paris : Harmattan, 2010 : 209-220.

Deslandres, Paul. Le Théâtre de la Foire à Paris pendant la première moitié du XVIII siècle. Paris : Picard, 1901, extrait de la «Revue des études historiques », mai-juin 1901.

Dictionnaire de l'Académie française. Paris : Coignard. Édition consultée : 1762.

Parfaict, Claude et Godin d'Abguerbe. Dictionnaire des théâtres de Paris. Paris : Rozet, 1767, rééd. Genève, Slatkine, 1967.

Gazetins de la Police secrète. Paris : Bibliothèque de 1'Arsenal, Ms-10155.

Le Sage et d'Orneval. Le Théâtre de la foire ou l'Opéra-Comique contenant les meilleures pièces qui ont été représentées aux foires de Saint-Germain et de Saint-Laurent, Paris : Ganeau, 1724, Pissot, 1728, Ganouin, 1731-1737, rééd. Genève : Slatkine, 1968.

Chevalier de Mouhy. Abrégé de l'histoire du théâtre français depuis son origine jusqu'à l'année 1780. Paris : chez l'auteur, Jorry, Mérigot, 1780, t. II.

Parfaict, Claude et François. Mémoires pour servir à l'histoire des spectacles de la Foire, par un acteur forain. Paris : Briasson, 1743.

Piron, Alexis. Les Fêtes de l'hymen ou La Rose. Bruxelles : np, 1752.

Porot, Bertrand. Le premier opéra-comique : histoire institutionnelle et artistique. Np : Vrin, collection «MusicologieS », à paraître.

---, «Aux origines de l'opéra-comique : étude musicale du Théâtre de la Foire de Lesage et d'Orneval (1713-1734)». The Opera-comique in the Eighteenth and Nineteenth 
Centuries. Dir. Lorenzo Frassa. Turnhout: Brepols (Speculum Musicae 15), 2011 : 283-329.

Quinault, Philippe. Atys, tragédie en musique ornée d'entrées de ballet, de machines et de changements de théâtre. Paris : Ballard, 1676.

Sakhnovskaia-Pankeeva, Anastasia. "La naissance des théâtres de la Foire : influence des Italiens et constitution d'un répertoire », thèse de doctorat, dir. Françoise Rubellin, U de Nantes, 2013. Non publiée.

\footnotetext{
Notes

${ }^{1}$ Nous préparons, dans le cadre de notre thèse «Contrainte et inventivité, de la guerre des théâtres à l'avènement d'un nouveau répertoire », une analyse détaillée de l'histoire du théâtre de la Foire des années 1717 à 1727. Pour une étude détaillée des années 1697 à 1716, voir Anastasia Sakhnovskaia.

${ }^{2}$ TFLO, t. I, p. vi, frontispice représentant la scène 8 de l'acte III d'Arlequin roi de Sérendib.

${ }^{3}$ Voir Les funérailles de la Foire de Le Sage et d'Orneval, TFLO, t. III.

${ }^{4}$ Voir Mercure, juillet 1725, p. 1655 et août 1725, p. 1852.

${ }^{5}$ Pauline Beaucé propose une étude approfondie des parodies d'opéra au dix-huitième siècle dans Parodies d'opéra au siècle des Lumières : évolution d'un genre comique.

${ }^{6}$ Voir Beaucé, «Les jeux de mots dans le répertoire des théâtres de la Foire à Paris au XVIII ${ }^{\mathrm{e}}$ siècle ».

${ }^{7}$ Voir Françoise Rubellin, « Airs populaires et parodies d'Opéra : jeux de sens dans les vaudevilles aux théâtres de la Foire et à la Comédie-Italienne », dans L'invention des genres lyriques français et leur redécouverte au XIX $X^{e}$ siècle, p. 163-176. Beaucé propose elle aussi une typologie des vaudevilles dans Parodies d'opéra au siècle des Lumières.

${ }^{8}$ Rubellin, dans Le Théâtre de la Foire : Anthologie de pièces inédites (1712-1726). Montpellier : Espaces 34, 2005, p. 43-44, en examine les différents objets.
} 